\title{
Validación de la versión en español del Cuestionario de Metas de Amistad en Educación Física*
}

\author{
Validation of the Spanish Version of the Friendship \\ Goals Questionnaire in Physical Education
}

Recibido: septiembre 24 de 2012 | Revisado: abril 21 de 2013 | Aceptado: octubre 24 de 2013

\author{
ANTONIO MÉNDEZ-GIMÉNEZ ** \\ JAVIER FERNÁNDEZ-RÍO \\ José-ANTONIO CECCHINI-EsTRADA \\ Universidad de Oviedo, España
}

doi:10.11144/Javeriana.UPSY13-1.vvec

Para citar este artículo: Méndez-Giménez, A., Fernández-Río, J. \& Cecchini-Estrada, J. -A. (2014). Validación de la versión en español del Cuestionario de Metas de Amistad en Educación Física. Universitas Psychologica, 13(1), 227-237. doi:10.11144/ Javeriana.UPSY13-1.vvec

* Artículo de investigación.

** Facultad de Formación del Profesorado y Educación. C/ Aniceto Sela, s/n. Despacho 239. Oviedo. 33005 Tlno. 985102850, Fax. 985103226. E-mails: mendezantonio@uniovi.es, javier.rio@ uniovi.es, cecchini@uniovi.es

\section{R E S U MEN}

De manera análoga a lo acontecido en la motivación de logro, el marco de aproximación-evitación ha sido incorporado al ámbito de la motivación social. El objetivo del estudio fue validar al castellano el Relationship Goals Questionnaire-Friendship Version de Elliot, Gable y Mapes (2006), en el contexto de la Educación Física. La muestra estuvo formada por un total de 273 adolescentes españoles (143 varones y 130 mujeres) con edades comprendidas entre 12-17 años de edad. El Análisis Factorial Confirmatorio apoyó la estructura bifactorial de las metas de amistad. Se comprobaron las propiedades psicométricas del cuestionario, y los resultados revelaron una validez y consistencia interna satisfactorias. Este instrumento es clave para investigar la motivación desde una perspectiva holística. Se sugieren líneas de investigación futuras.

Palabras clave autores

Análisis factorial confirmatorio, Educación Física, metas sociales,

Palabras clave descriptores

Psicología del deporte, investigación cuantitativa, psicometría.

\section{A B S T R A C T}

The approach-avoidance framework has been incorporated into the social motivation context, as it happened before in the achievement goals. The aim of this research work was to validate for Spanish Physical Education contexts the Elliot, Gable and Mapes (2006) Relationship Goals QuestionnaireFriendship Version. The sample consisted of 273 Spanish adolescents (143 males, 130 females) $12-17$ years old. Confirmatory Factor Analysis supported the two-factor structure of the friendship goals. Psychometric properties of the questionnaire were tested, and results showed acceptable validity and internal consistency. This instrument is a key element to investigate motivation from a holistic point of view. Future lines of research are suggested. Keywords authors

Confirmatory factor analysis, physical education, social goals, psychometry.

Keywords plus

Sport psychology, quantitative research, psychometry. 
Frecuentemente, la motivación es definida como 'energización' y 'dirección' de la conducta (p. ej., Heckhausen, 1991). Por lo general, uno de los marcos teóricos más empleado para examinar la motivación, tanto de logro como social, es la Teoría de Metas (p. ej., Dweck, 1986; Elliot \& Church, 1997; Guan, Xiang, McBride \& Bruene, 2006; Nicholls, 1984). Si bien, tradicionalmente, la investigación sobre los aspectos sociales de la motivación ha sido escasa, en los últimos años cada vez más académicos han reclamado la necesidad de incluir la motivación social en la investigación motivacional y dirigir este campo hacia la perspectiva de metas múltiple (Allen, 2003; Elliot, Gable \& Mapes, 2006; King \& McInerney, 2012; Valle-Arias et al., 2010). En un principio, las dos metas de logro propuestas se distinguieron de acuerdo a la definición de la competencia (Elliot, 1999) y recibieron diferentes denominaciones: metas de maestría y metas de rendimiento, según Dweck (1986), o metas de implicación a la tarea y de implicación al ego, siguiendo a Nicholls (1984). En este marco dicotómico, las metas de maestría (implicación a la tarea) se refieren al desarrollo de la competencia a través de la maestría de la tarea, mientras que las metas de rendimiento (implicación al ego) se representan como demostración de competencia en relación con los demás.

Elliot y McGregor (2001), además de comprender la definición de la competencia, incluyeron una segunda dimensión en el desarrollo del marco de metas de logro 2x2: la dimensión de la valencia. Así, la competencia podría ser definida de dos formas: por un lado según la definición: absoluta/ intrapersonal (maestría) vs. normativa (rendimiento), por otro, en función de la valencia: positiva (aproximación) o negativa (evitación). La motivación de aproximación implica la energización o la dirección de la conducta hacia estímulos positivos (metas, acontecimientos o posibilidades), mientras que la motivación de evitación implica la energización o dirección del comportamiento alejándose de estímulos negativos (Elliot, 2006). Las metas resultantes derivan de la combinación de ambas dimensiones fundamentales: metas de aproximación-maestría, evitación-maestría, aproximaciónrendimiento y evitación rendimiento.
En las últimas décadas se han definido y examinado diversos tipos de metas sociales, como el bienestar, la responsabilidad y la afiliación (Urdan \& Maerhr, 1995), la responsabilidad y las relaciones: amistad y aceptación del grupo (Patrick, Hicks \& Ryan, 1997), la afiliación, el estatus y el reconocimiento (Allen, 2003), o la amistad, la aceptación del grupo y los elogios del entrenador (Stuntz \& Weiss, 2003). Sin embargo, las medidas de metas sociales que en la actualidad se centran en valencias de aproximaciónevitación aún son muy limitadas (Elliot et al., 2006; Gable, 2006; Ryan \& Shim, 2006). Esta limitación es sorprendente puesto que la distinción aproximaciónevitación es una idea muy antigua en la historia del pensamiento psicológico, y sus comienzos en el análisis de la motivación y la conducta se remonta a finales del siglo XIX (Elliot, 2006).

Elliot et al. (2006) incorporaron el marco de aproximación-evitación al ámbito de la motivación social, específicamente, en la motivación de amistad. Las metas de aproximación-amistad se caracterizan por el esfuerzo de competencia social, mientras que las metas de evitación-amistad se definen por el esfuerzo para evitar la incompetencia social (Elliot et al., 2006). Las metas sociales de aproximación y evitación son representaciones cognitivas de nivel inferior que dirigen a los individuos, respectivamente, hacia potenciales resultados relacionales positivos (por ejemplo, tratar de profundizar en las relaciones personales) o les alejan de los posibles resultados relacionales negativos (por ejemplo, tratar de evitar conflictos relaciones).

Elliot et al. (2006) desarrollaron el Cuestionario de Metas de Amistad (Relationship Goals Questionnaire-Friendship [RGQ-F]) con medidas de metas de aproximación-amistad y de metas de amistad-evitación. En un primer estudio, Elliot y sus colaboradores informaron del desarrollo de medidas consistentes internamente, con propiedades psicométricas sólidas y correlaciones moderadas $(r=0.4)$ entre las metas de aproximación-amistad y evitación-amistad. Además de una adecuada fiabilidad y validez, las medidas desarrolladas fueron consideradas como bastante económicas puesto que solo se componen de cuatro ítems por escala. Un análisis factorial exploratorio (en un primer estudio) y el análisis factorial confirmatorio 
(segundo estudio) apoyaron la estructura bifactorial de los ítems, proporcionando apoyo adicional a los datos de medida. En un segundo estudio, los investigadores encontraron que las metas de aproximaciónamistad fueron un predictor positivo de la satisfacción de la relación y de la frecuencia de acontecimientos relacionales positivos (por ejemplo, buenas interacciones con los amigos). Además, se mostraron como predictor negativo de la soledad y de la frecuencia de los acontecimientos relacionales negativos (por ejemplo, sentirse excluido o rechazado por un amigo o miembro de la familia). Las metas de evitaciónamistad, por otra parte, fueron un predictor positivo de la soledad, de la frecuencia de los acontecimientos relacionales negativos y del impacto de los acontecimientos relacionales negativos.

Garn y Sun (2009) aplicaron la versión Amistad del RGQ de Elliot et al. (2006) en el contexto de la Educación Física (EF) entre los adolescentes estadounidenses, y concluyeron que la redacción del texto se ajustaba bien al nivel de comprensión lectora de la población en esta franja de edad. Los resultados del análisis factorial confirmatorio indicaron una validez de constructo aceptable a nivel estructural. En otro orden de cosas, las metas sociales se han relacionado con las metas de logro tanto en el contexto académico (Elliot et al., 2006), como en el ámbito deportivo (Hodges, Allen \& Smellie, 2008; Stuntz \& Weiss, 2009) y en entorno de la EF (Garn \& Sun, 2009; Méndez-Giménez, Fernández-Río \& Cecchini, 2012).

Garn, Ware y Solomon (2011) enfatizaron las peculiaridades de la estructura y organización de las clases de EF respecto a las demás aulas tradicionales. Con frecuencia, la estructura de una clase académica tradicional permite a los estudiantes un ambiente privado para completar las tareas y restringe las oportunidades sociales, porque los estudiantes pasan sentados la mayor parte del tiempo e interactúan en ocasiones limitadas unos con otros. Además, en el contexto de clase tradicional también son comunes las actividades dirigidas por el profesor. En comparación, las clases de EF generalmente se centran en la mejora de habilidades físicas y a menudo están relacionadas con actividades deportivas. Esto genera una dinámica distinta, de una interacción profesor-alumno se pasa a una mayor interacción alumno-alumno. En palabras de Garn et al. (2011), las clases de EF tienden a ser públicas (al menos de cara al grupo de compañeros) y a estar basadas en el rendimiento, lo que amplifica los cambios autoconscientes que experimentan los adolescentes. En consecuencia, los adolescentes tienden a acercarse a los ambientes de actividad física con múltiples metas sociales y de logro (Solmon, 2006).

Los estudios de Garn y Sun (2009) y MéndezGiménez, Fernández-Río y Cecchini (2012) han explorado la influencia que ejercen las metas sociales y de logro (aproximación y evitación), por separado y conjuntamente, sobre diversas consecuencias motivacionales en los estudiantes de EF. Ambos trabajos pusieron de relieve la necesidad de incluir las metas sociales de amistad (aproximación-evitación) en los estudios motivacionales. Además, los resultados del estudio de Méndez-Giménez et al. (2012) mostraron que las metas de aproximación-maestría y evitaciónamistad fueron los únicos predictores positivos del esfuerzo, mientras que las metas de aproximaciónmaestría y aproximación-amistad fueron los únicos predictores positivos de la diversión.

Teniendo en cuenta el interés de la distinción aproximación-evitación en el ámbito social, las peculiaridades estructurales y organizativas de las clases de EF y su influencia en los aspectos sociales en los alumnos adolescentes, en este estudio el propósito fue validar al castellano el Cuestionario de Metas de Amistad en Educación Física (RGQ-F) de Elliot et al. (2006), adaptándolo a la población española adolescente. En ese sentido, se decidió emplear una escala Likert de 5 puntos, en lugar de la de 7 puntos utilizada con estudiantes universitarios en el estudio original, con el objeto de simplificar las opciones de respuesta y proporcionar un referente de medida más próximo al asumido habitualmente en el contexto educativo español.

\section{Método}

\section{Participantes}

La muestra estuvo formada por un total de 273 jóvenes (143 varones y 130 mujeres) de un institu- 
to del norte de España con edades comprendidas entre los 12 y 17 años de edad $(M=14.29, D E$ $=1.68)$. El tipo de muestreo fue aleatorio simple. Bentler (1990) sugirió que el tamaño muestral en un análisis factorial confirmatorio debe mantener una ratio de 5:1 sobre los parámetros libres, si bien lo más adecuado es contar, al menos, con 10 sujetos por parámetro (Nunnally, 1978). En referencia a tamaños muestrales específicos, Anderson y Gerbing (1984) recomendaron el uso de una muestra de alrededor 200 sujetos cuando existen al menos tres indicadores por variable latente. En este caso los parámetros libres estimados son 17, por lo que la muestra fue considerada adecuada.

\section{Instrumentos}

\section{Metas de amistad}

Se empleó la traducción al castellano del RGQ-F validado por Elliot et al. (2006) en el ámbito académico y aplicado en el contexto de la EF por Garn y Sun (2009) con niveles de fiabilidad y consistencia interna satisfactorios. Esta escala está compuesta por un total de 8 ítems agrupados en dos factores: Metas de Aproximación-Amistad (4 ítems), que miden los esfuerzos por lograr competencia social (p. ej., "En las clases de EF trato de profundizar en las relaciones con mis amigos") y Metas de EvitaciónAmistad (cuatro ítems), que miden los esfuerzos por evitar la incompetencia social (p. ej., "En las clases de EF trato de evitar desacuerdos y conflictos con mis amigos"). Los alfas de Cronbach originales en el trabajo de Garn y Sun (2009) fueron 0.88 y 0.87 para la Metas de Aproximación-Amistad y Evitación-Amistad, respectivamente.

\section{Metas sociales}

Para evaluar las metas sociales de los estudiantes se empleó la Escala de Meta Social de 11 ítems de Patrick et al. (1997), adaptada al contexto de la EF por Guan et al. (2006) (Social Goal Scale-PE). Las Metas de Relación se refieren al deseo del estudiante de formar y mantener relaciones positivas entre los compañeros del centro educativo (Patrick et al.,
1997) y las Metas de Responsabilidad social representan el deseo de adherirse a las normas sociales y a las expectativas de los roles (Wentzel, 1991, 1993). Moreno, González-Cutre y Sicilia (2007) tradujeron y validaron esta escala al español al analizar sus propiedades psicométricas y comprobar su consistencia en la población estudiantil española. Seis ítems abordan las Metas de Relación (por ejemplo, "Me gustaría llegar a conocer realmente bien a mis amigos del colegio") y cinco ítems abordan las Metas de Responsabilidad (por ejemplo, "Intento hacer lo que el profesor me pide”). Los alfas de Cronbach originales fueron 0.82 y 0.76 para la Meta de Responsabilidad y la de Relación, respectivamente. Se han encontrado correlaciones de moderadas a altas entre las Metas de Relación y Responsabilidad en el contexto de la EF $(r=0.24,0.54,0.88$ en los estudios de Cecchini et al., 2008; Guan et al., 2006; Moreno et al., 2007, respectivamente).

\section{Procedimiento}

En primer lugar, se realizó una traducción doble (traducción-retrotraducción) de los ítems del Cuestionario de Metas de Amistad de Elliot et al. (2006) adaptándolo al contexto de la EF tal y como ya habían hecho previamente Garn y Sun (2009). Para facilitar la lectura y comprensión, se evitaron estructuras repetitivas en la redacción y se introdujo la siguiente raíz común a todos los ítems: "En mis clases de Educación Física trato de..." (en el Apéndice 1 puede encontrarse la versión definitiva). Dos expertos supervisaron que se mantuviera una estrecha similitud con la escala original (Hambleton, 1996). A partir de ese momento, se solicitó el permiso del Comité de Ética de la Universidad donde se realizó el estudio, así como del centro educativo participante. Igualmente, se obtuvo el consentimiento informado de los padres de todos los participantes. Los estudiantes completaron de forma anónima los cuestionarios empleando aproximadamente unos 20 minutos. Para reducir al mínimo la tendencia a dar respuestas socialmente deseables, los encuestadores animaron a los estudiantes a que contestaran lo más verazmente posible y les aseguraron que sus respuestas no afectarían a 
sus notas de EF. Los datos fueron introducidos y analizados mediante los programas informáticos SPSS 19.0. y AMOS 18.0.

\section{Resultados}

\section{Análisis factorial confirmatorio}

Para la evaluación del modelo, se emplearon los siguientes índices de ajuste absoluto: la razón de verosimilitud $\chi^{2}$, la razón chi cuadrado/grados de libertad $\left(\chi^{2} / g l\right)$, el GFI (Goodness of Fit Index), RMSR (Root Mean Square Residual), RMSEA (Root Mean Square Error of Aproximation). Estos índices determinan el grado en el que el modelo predice, a partir de los parámetros estimados, la matriz de covarianzas observadas. También se utilizaron los siguientes índices de ajuste incremental: el IFI (Incremental Fit Index), NFI (Normed Fit Index), el CFI (Comparative Fit Index). Estos índices comparan el ajuste global del modelo propuesto con un modelo de referencia, habitualmente un modelo nulo en el que no se especifica ninguna relación entre las variables. Una probabilidad de $\chi^{2}$ superior a 0.05 indicaría un buen ajuste (Jöreskog \& Sörbom, 1993), pero debido a que por sí solo este índice es especialmente sensible al tamaño muestral se considera un ajuste aceptable si la razón $\chi^{2} / g l$ es inferior a 3 (Schermelleh-Engel, Moosbrugger \& Müller, 2003). Hu y Bentler (1999) sugirieron un corte estricto de 0.95 o superior en el IFI, NFI, GFI y el CFI, un valor de corte de 0.06 o por debajo de RMSEA y un valor de corte de 0.08 o por debajo para el RMSR. Sin embargo, los autores indicaron

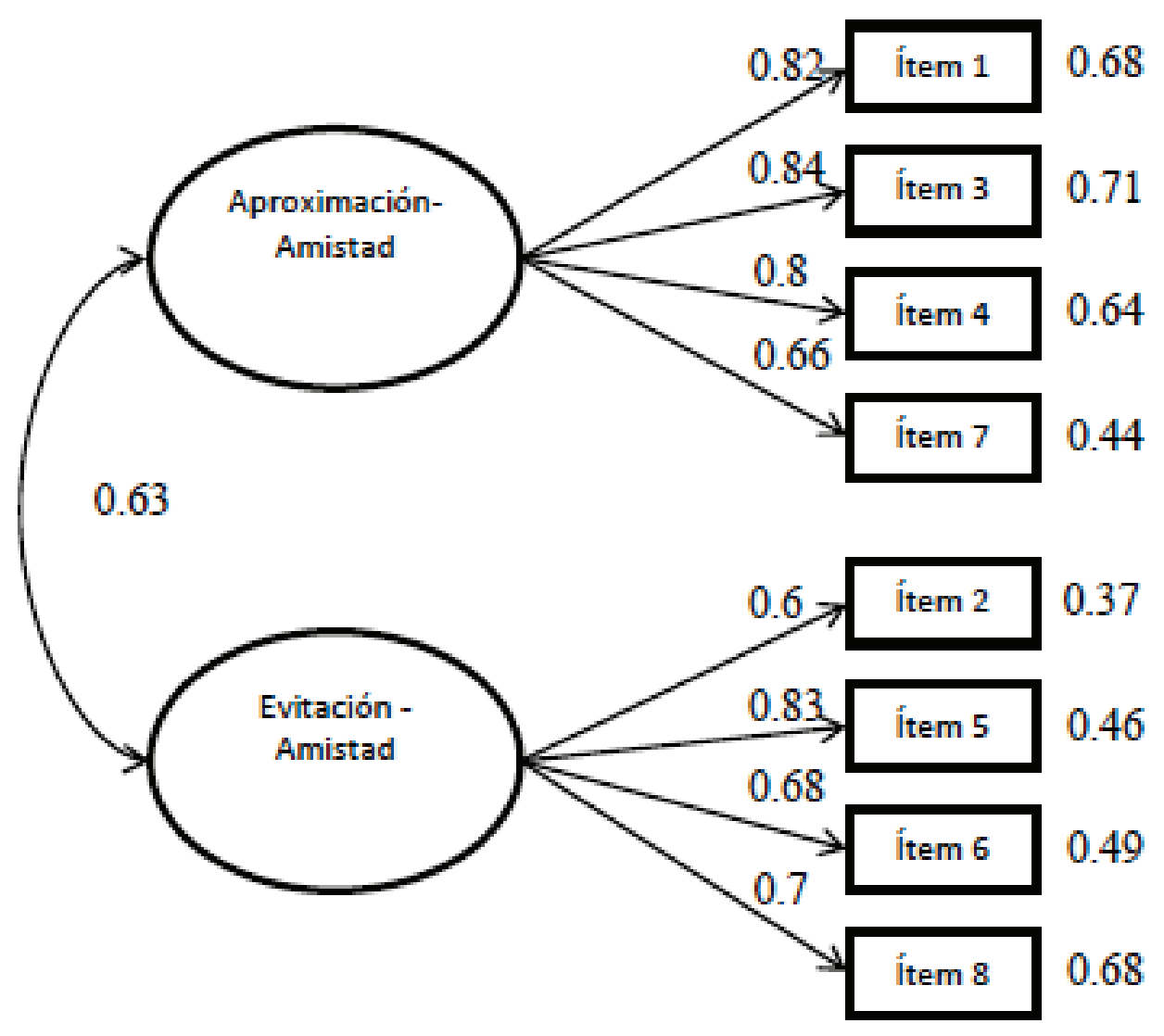

Figura 1. AFC del Cuestionario de Metas de Amistad en Educación Física.

Nota. Las elipses representan los factores y los rectángulos los diferentes ítems. Todos los parámetros están estandarizados y son significativos $(p<0.01)$.

Fuente: elaboración propia. 
que las reglas que se propusieron no debían generalizarse demasiado, ya que se basan en resultados de simulación de modelos en ciertas condiciones.

El análisis factorial confirmatorio (AFC) es una técnica de modelado de ecuaciones estructurales que asume la normalidad multivariada. Por lo tanto, el análisis inicial examinó la normalidad multivariante de los 8 indicadores. Los resultados del coeficiente de curtosis multivariada fue de 42.38, lo que indicó que las muestras presentaban una distribución no normal (Mardia, 1974). De acuerdo con Bollen y Stine (1992), las técnicas de bootstrapping pueden representar un medio ideal para abordar los problemas en situaciones en que no se cumplen los datos de una escala continua y con distribución normal multivariada. El BollenStine Bootstrap proporciona un valor $p$ sin asumir la normalidad de la muestra, con lo que corrige el valor probabilístico proporcionado por el método de máxima verosimilitud para contrastar el ajuste global. Se decidió hacer un remuestreo de 1.000 bootstraps, en función del tamaño de la muestra. Nevitt y Hancock (2001) señalaron que existía poca mejora en la calidad de las estimaciones del bootstrap por el hecho de hacer un gran número de remuestreos. Si el p-value por el Bollen-Stine Bootstrap fuera menor que 0.05 , entonces se rechazaría el modelo. Los intervalos de confianza de los pesos de regresión y los pesos de regresión estandarizados mostraron que los valores estimados eran significativamente diferentes de cero ( $p=0.083$; Byrne, 2001), por lo que debía ser aceptado. La Figura 1 muestra el AFC.
Los índices de ajuste mostraron que la hipótesis del modelo se ajusta razonablemente bien a los datos, $\chi^{2}(19)=38.35, p<0.01 ; \chi^{2} / g l=2.01 ; \mathrm{GFI}=0.96$; $\mathrm{IFI}=0.98 ; \mathrm{NFI}=0.96 ; \mathrm{CFI}=0.98 ; \mathrm{RMSEA}=$ $0.06 ; \operatorname{RMSR}=0.03$.

\section{Validez convergente y fiabilidad de las medidas}

La validez convergente se valoró mediante la significación estadística de las cargas factoriales de los indicadores de cada constructo latente. En la Tabla 1 puede observarse como todas las cargas estandarizadas y los coeficientes críticos (CR) superaron con amplitud los niveles mínimos recomendados de 0.5 y $1.96(p<0.05)$, respectivamente (Hair, Black, Babin, Anderson \& Tatham, 2006). La fiabilidad de las medidas se calculó mediante el coeficiente de fiabilidad compuesta (CFC) que, al no depender del número de atributos asociados, resulta más adecuado que el alfa de Cronbach (Cortina, 1993; Hair et al., 2006). Según Nunnally (1978), el valor mínimo que se considera apropiado para este coeficiente es 0.7. La varianza media extraída (Average Variance Extracted [AVE]) representa la cantidad total de la varianza de los indicadores recogida por la variable latente. Cuanto mayor sean los valores de AVE, más representativos son los indicadores de la variable latente en la que cargan. Generalmente, se recomienda que el valor de AVE sea superior 0.5 (Bagozzi \& Yi, 1988; Hair et al., 2006). Las dos pruebas de fiabilidad sobrepasaron los valores aceptables, tanto

\section{TABLA 1}

Cargas factoriales $(\lambda)$, coeficientes críticos (CR), coeficiente de fiabilidad compuesta (CFC) y varianza media extraída (AVE)

\begin{tabular}{lccccc}
\hline & Items & $\lambda$ & CR & CFC & AVE \\
\hline \multirow{3}{*}{ Aproximación-amistad } & Ítem 1 & 0.82 & - & 0.86 & 0.62 \\
& Ítem 3 & 0.84 & $15.630^{*}$ & & \\
& Ítem 4 & 0.8 & $14.331^{*}$ & & \\
& Ítem 7 & 0.66 & $11.158^{*}$ & & \\
\hline \multirow{3}{*}{ Evitación-amistad } & Ítem 2 & 0.6 & - & 0.8 & 0.5 \\
& Ítem 5 & 0.83 & $9.588^{*}$ & & \\
& Ítem 6 & 0.68 & $8.661^{*}$ & & \\
& Ítem 8 & 0.7 & $8.520^{*}$ & & \\
\hline
\end{tabular}

$* p<0.001$.

Fuente: elaboración propia. 
en la subescala de Aproximación-Amistad como en la de Evitación-Amistad (Tabla 1).

\section{Validez discriminante}

Se comparó la raíz cuadrada de la AVE con la correlación entre ambos constructos (Fornell \& Larcker, 1981). En la Tabla 2 se presenta esta correlación y, en la diagonal, la raíz cuadrada de la AVE. Según los autores para que exista validez discriminante entre constructos es necesario que la raíz cuadrada de la AVE sea superior a la correlación entre los mismos. Considerando estos resultados, se puede afirmar que existe una validez discriminante entre los constructos, si bien, ambos están bastante relacionados.

\section{Validez concurrente}

Se calcularon las medias para las dos metas sociales (relación y responsabilidad) y se realizaron dos análisis de regresión lineal (entry), empleando dichas metas como variables dependientes y las metas de aproximación-amistad y evitación-amistad como variables independientes. En cuanto a las correlaciones entre las metas sociales (responsabilidad y relación) y las metas de amistad, se encontraron correlaciones positivas de medias a moderadas en todas ellas. No obstante, la única variable predictora la responsabilidad fueron las metas de evitaciónamistad $(\beta=36)$, mientras que en el caso de la relación tanto las metas las metas de aproximaciónamistad $(\beta=22)$ como las de evitación amistad $(\beta=47)$ fueron predictoras. Las metas de amistad explicaron el $17 \%$ de la varianza de las metas de responsabilidad y el 39\% de la varianza de las metas de relación (Tabla 3).

\section{Discusión}

El objetivo de este trabajo fue validar en castellano el Cuestionario de Metas de Amistad de Elliot et al. (2006) adaptándolo al contexto de la EF. Esta validación se ha realizado tanto en lo que se refiere a la estructura factorial del cuestionario como a las relaciones de las metas de amistad con otras variables con las que se supone que dichas metas deben estar relacionadas: las metas de responsabilidad y de relación social (validez concurrente).

En relación con la estructura factorial del cuestionario, de manera consistente con los hallazgos informados por Elliot et al. (2006) y Garn y Sun (2009), los análisis realizados confirman la existencia de un modelo de dos factores de metas de

\section{TABLA 2}

Correlación entre variables y validez discriminante

\begin{tabular}{lcc}
\hline & 1 & 2 \\
\hline 1. Aproximación-amistad & 0.79 & \\
2. Evitación-amistad & 0.63 & 0.71 \\
\hline
\end{tabular}

Nota. Todas las correlaciones son significativas $(p<0.01)$. La raíz cuadrada del AVE se muestra en la diagonal. Fuente: elaboración propia.

\section{TABLA 3}

Análisis de regresión lineal utilizando las metas de responsabilidad y de relación como variables dependientes y las metas de amistad (aproximación-evitación) como variables independientes

\begin{tabular}{lcccc}
\hline & \multicolumn{2}{c}{ Responsabilidad } & \multicolumn{2}{c}{ Relación } \\
& $\mathrm{r}$ & $\beta$ & $\mathrm{r}$ & $\beta$ \\
\hline Aproximación-Amistad & $0.27^{*}$ & 0.08 & $0.48^{*}$ & $0.22^{*}$ \\
Evitación-Amistad & $0.41^{*}$ & $0.36^{*}$ & $0.6^{*}$ & $0.47^{*}$ \\
$\mathrm{R}^{2}$ & & $0.17^{*}$ & & $0.39^{*}$ \\
\hline
\end{tabular}

Nota. $r=$ Correlación de Pearson, $\beta=$ Beta estandarizada, $R=$ Coeficiente de Correlación Múltiple. $* p<0.001$.

Fuente: elaboración propia. 
amistad en el contexto de la EF. Las puntuaciones de los factores Aproximación-Amistad y EvitaciónAmistad exhibieron propiedades de validez psicométrica favorables. Todos los índices fueron aceptables, lo que indica que el Cuestionario de Metas de Amistad produce puntuaciones válidas y que cada meta de amistad representaba a un constructo distinto. Los ítems mostraron cargas relativamente fuertes $(0.84$, la mayor y 0.6 , la menor) y valores de $t$ satisfactorios. Así mismo, los coeficientes de fiabilidad compuesta (CFC) y de varianza media extraída (AVE) resultaron apropiados.

La correlación encontrada entre ambos constructos de metas de amistad en nuestro estudio es muy similar a la informada en el mismo contexto de EF por Garn y Sun (2009), siendo de 0.63 y 0.69, respectivamente. Dichas asociaciones son algo más fuertes que las mostradas en el contexto académico por Elliot et al. (2006), si bien estas últimas fueron obtenidos con una muestra de estudiantes universitarios (Producto Momento de Pearson $=0.4$ ). Las diferencias contextuales (académico - EF) y de edad (adolescentes - jóvenes universitarios) podrían explicar estas diferencias. No obstante, investigaciones futuras podrán ayudar a explicar mejor las causas de estas diferencias.

En cuanto a las correlaciones entre las metas sociales y las metas de amistad, se encontraron correlaciones positivas de medias a moderadas en todas ellas, lo que fue consecuente con los resultados de validación externa esperados. Las relaciones más altas se dieron entre las metas de amistad y las metas de relación, explicando las primera el 39\% de la varianza de las segundas. Las metas de relación, definidas en el estudio de Guan et al. (2006) como el deseo de un individuo de formar y mantener relaciones positivas con los compañeros de clase, tienen muchas semejanzas con las metas sociales de amistad definidas por Elliot et al. (2006). Gable y sus colegas (Elliot et al., 2006; Gable, 2006) desarrollaron y proporcionado evidencia empírica de la distinción aproximación-evitación en el ámbito social con el desarrollo de metas de aproximación y las metas de evitación a la amistad. Las metas de aproximación a la amistad se caracterizan por los esfuerzos realizados para lograr competencia social, mientras que las metas de evitación de la amistad se definen como evitación de incompetencia social. De manera interesante, esta distinción aproximación-evitación en la motivación social y la validación de instrumento de medida al castellano ofrecen un nuevo marco para explorar la conexión entre metas de logro y las metas sociales desde la perspectiva más integral, así como sus relaciones con resultados motivacionales.

En conclusión, los investigadores y profesores de EF pueden encontrar en este trabajo una herramienta en español, el Cuestionario de Metas de Amistad, válida y fiable, específica del contexto de la EF, que permite identificar las dos distinciones de las metas sociales de amistad: aproximación y evitación. El empleo de las valencias positivas y negativas en el campo de la motivación social viabiliza un análisis más profundo de la motivación en las clases de EF y, por ende, puede contribuir al avance en el conocimiento de los principios que dirigen y energizan la conducta, y que provocan una mayor implicación en la práctica físico-deportiva y, por extensión, en la adopción de estilos de vida más saludables. Este cuestionario ha sido probado con la población adolescente, un periodo crítico de la vida en el que la calidad de las relaciones de amistad desempeña un papel crucial en el desarrollo personal de los estudiantes.

No obstante, es sabido que la validación de todo instrumento de medidas y escalas conlleva un proceso continuo de comprobación y precisa de nuevos análisis de las propiedades psicométricas en otros contextos similares. Además, nuevos estudios deberían comprobar la invarianza a través del género y las correlaciones con otras variables y consecuencias motivacionales. La inclusión de la doble valencia (aproximación-evitación) traza un camino a seguir con otros constructos y medidas sociales (como la relación estudiante-docente o la responsabilidad de los estudiantes en clase), y fortalece la vía de investigación sobre las interacciones de aproximación-evitación entre las metas de logro y sociales desde la perspectiva de metas múltiples. Por otro lado, la investigación debería considerar el tipo de actuación docente (clima motivacional) para desarrollar las metas sociales que llevan a consecuencias más adaptativas. 


\section{Referencias}

Allen, J. B. (2003). Social motivation in youth sport. Journal of Sport E⿱ Exercise Psychology, 25(4), 551567.

Anderson, J. C. \& Gerbing, D. W. (1988). Structural equation modeling in practice: A review and recommended two-step approach. Psychological Bulletin, 103(3), 411-423.

Bagozzi, R. P. \& Yi, Y. (1988). On the evaluation of structural equation models. Journal of the Academy of Marketing Science, 16(2), 74-94.

Bentler, P. M. (1990). Comparative fit indexes in structural models. Psychological Bulletin, 107(2), 238246.

Bollen, K. A. \& Stine, R. A. (1992). Bootstrapping goodness-of-fit measures in structural equation models. Sociological Methods EO Research, 21(2), 205-229.

Byrne, B. M. (2001). Structural equation modeling with AMOS: Basic concepts, applications and programming. Mahwah, NJ: Lawrence Erlbaum Associates.

Cecchini, J. A., González, C., Méndez, A. FernándezRío, J., Contreras, O. \& Romero, S. (2008). Metas sociales y de logro, persistencia-esfuerzo e intenciones de práctica deportiva en el alumnado de Educación Física. Psicothema, 20(2), 260-265.

Cortina, J. M. (1993). What is coefficient alpha? An examination of theory and applications. Journal of Applied Psychology, 78(1), 98-104.

Dweck, C. S. (1986). Motivational processes affecting motivation. The American Psychologist, 41(10), 1040-1048.

Elliot, A. J. (1999). Approach and avoidance motivation and achievement goals. Educational Psychologist, 34(3), 149-169.

Elliot, A. J. (2006). The hierarchical model of approachavoidance motivation. Motivation and Emotion, 30(2), 111-116.

Elliot, A. J. \& Church, M. A. (1997). A hierarchical model of approach and avoidance achievement motivation. Journal of Personality and Social Psychology, 72(1), 218-232.

Elliot, A. J., Gable, S. L. \& Mapes, R. R. (2006). Approach and avoidance motivation in the social domain. Personality and Social Psychology Bulletin, 32(3), 378-391.
Elliot, A. J. \& McGregor, H. A. (2001). A 2×2 achievement goal framework. Journal of Personality and Social Psychology, 80(3), 501-519.

Fornell, C. \& Larcker, D. F. (1981). Evaluating structural equation models with unobservable variables and measurement error: Algebra and statistics. Journal of Marketing Research, 18(3), 39-50.

Gable, S. L. (2006). Approach and avoidance social motives and goals. Journal of Personality, 74(1), $175-222$.

Garn, A. C. \& Sun, H. (2009). Approach-Avoidance motivational profiles in early adolescents to the PACER fitness test. Journal of Teaching in Physical Education, 28(4), 400-421.

Garn, A. C., Ware, D. R. \& Solmon, M. A. (2011). Student engagement in high school physical education: Do social motivation orientations matter? Journal of Teaching in Physical Education, 30(1), 84-98.

Guan, J., Xiang, P., McBride, R. \& Bruene, A. (2006). Achievement goals, social goals, and students' reported persistence and effort in high school physical education. Journal of Teaching in Physical Education, 25(1), 58-74.

Hair, J. F., Black, W. C., Babin, B. J., Anderson, R. E. \& Tatham, R. L. (2006). Multivariate data analysis (6a. ed.). New Jersey: Pearson.

Hambleton, R. K. (1996). Adaptación de test para su uso en diferentes idiomas y culturas: fuentes de error, posibles soluciones y directrices prácticas. En J. Muñiz (Ed.), Psicometría (pp. 203-238). Madrid: Universitas.

Heckhausen, H. (1991). Motivation and action (P. Leppman, Trad.) New York: Springer-Verlag.

Hodges, K., Allen, J. B. \& Smellie, L. (2008). Motivation in masters sport: Achievement and social goals. Psychology of Sport y Exercise, 9(2), 157-176.

Hu, L. \& Bentler, P. M. (1999). Cutoff criteria for fit indexes in covariance structure analysis: Conventional criteria versus new alternatives. Structural Equation Modeling: A Multidisciplinary Journal, 6(1), 1-55.

Jöreskog, K. G. \& Sörbom, D. (1993). LISREL 8: Structural equation modeling with the SIMPLIS command language. Hillsdale, NJ: Lawrence Erlbaum Associates Publishers. 
King, R. B. \& McInerney, D. M. (2012). Including social goals in achievement motivation research: Examples from the Philippines. Online Readings in Psychology and Culture, Unit 5. Recuperado el 2 de mayo de 2012, de http://scholarworks.gvsu. edu/orpc/vol5/iss3/4

Mardia, K. V. (1974). Applications of some measures of multivariate skewness and kurtosis in testing normality and robustness studies. Sankhya: The Indian Journal of Statistic, 36(2), 115-128.

Méndez-Giménez, A., Fernández-Río, J. \& Cecchini, J. A. (2012). Análisis de un modelo multiteórico de metas de logro, metas de amistad y autodeterminación en Educación Física. Estudios de Psicología, 33(3), 325-336.

Moreno, J. A., González-Cutre, D. \& Sicilia, A. (2007). Metas sociales en las clases de Educación Física. Análisis y Modificación de Conducta, 33(149), 351368.

Nevitt, J. \& Hancock, G. R. (2001). Performance of bootstrapping approaches to model test statistics and parameter standard error estimation in structural equation modeling. Structural Equation Modeling: A Multidisciplinary Journal, 8(3), 353-377.

Nicholls, J. G. (1989). The competitive ethos and democratic education. Cambridge, MA: Harvard University Press.

Nunnally, J. C. (1978). Psychometric theory. New York: McGraw-Hill.

Patrick, H., Hicks, L. \& Ryan, A. M. (1997). Relations of perceived social efficacy and social goal pursuit to self-efficacy for academic work. Journal of Early Adolescence, 17(2), 109-128.

Ryan, A. M. \& Shim, S. S. (2006). Social achievement goals: The nature and consequences of different orientations toward social competence. Personality and Social Psychology Bulletin, 32(9), 1246-1263.

Schermelleh-Engel, K., Moosbrugger, H. \& Muüller, H. (2003). Evaluating the fit of structural equation models: Tests of significance and descriptive goodness-of-fit measures. Method of Psychological Research, 8(2), 23-74.

Solmon, M. A. (2006). Goal theory in physical education classes: Examining goal profiles to understand achievement motivation. International Journal of Sport and Exercise Psychology, 4(3), 325-346.

Stuntz, C. P. \& Weiss, M. R. (2003). Influence of social goal orientations and peers on unsportsmanlike play. Research Quarterly for Exercise and Sport, 74(4), 421-435.

Stuntz, C. P. \& Weiss, M. R. (2009). Achievement goal orientations and motivational outcomes in youth sport: The role of social orientations. Psychology of Sport and Exercise, 10(2), 255-262.

Urdan, T. C. \& Maehr, M. L. (1995). Beyond a two-goal theory of motivation and achievement: A case for social goals. Review of Educational Research, 65(3), 213-243.

Valle-Arias, A., Rodríguez, S., Cabanach, R. G., NúñezPérez, J. C., González-Pienda J. A. \& Rosario, P. (2010). Perfiles motivacionales y diferencias en variables afectivas, motivacionales y de logro. Universitas Psychologica, 9(1), 109-121.

Wentzel, K. R. (1991). Social competence at school: Relation between social responsibility and academic achievement. Review of Educational Research, 61(1), $1-24$.

Wentzel, K. R. (1993). Motivation and achievement in early adolescence: The role of multiple classroom goals. Journal of Early Adolescence, 13(1), 4-20. 


\section{Apéndice 1}

\section{Cuestionario de Metas de Amistad en Educación Física}

$1=$ Totalmente en desacuerdo; 2 = En desacuerdo; 3 Indiferente 4 = De acuerdo; 5 = Totalmente de acuerdo

$$
\text { En mis clases de Educación Física trato de... }
$$

1. Profundizar en las relaciones con mis amigos $\quad \begin{array}{lllll}1 & 2 & 3 & 4 & 5\end{array}$

2. Evitar desacuerdos y conflictos con mis amigos $\quad \begin{array}{ccccccc}1 & 2 & 3 & 4 & 5\end{array}$

3. Mejorar y ampliar mis amistades $\quad \begin{array}{rlllll}1 & 2 & 3 & 4 & 5\end{array}$

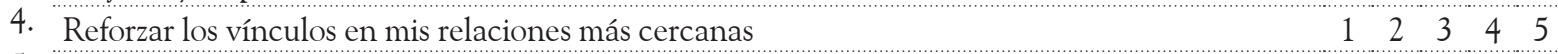

5. Evitar situaciones que podrían dañar mis amistades $\quad \begin{array}{llllllllll}1 & 2 & 3 & 4 & 5\end{array}$

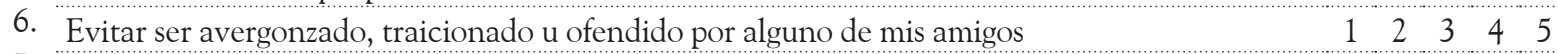

7. Compartir muchas experiencias divertidas y significativas con mis amigos $\quad \begin{array}{lllllllllllllll} & 2 & 3 & 4 & 5\end{array}$

8. Asegurarme de que no le pase nada malo a mis amistades más cercanas $\quad \begin{array}{lllll}2 & 3 & 4 & 5\end{array}$

Aproximación-Amistad: 1, 3, 4, 7

Evitación-Amistad: 2, 5, 6, 8 
